Slaughter, . (2000). Interfaces for understanding: Improving access to consumer health information. . 11th ASIS SIG/CR Classification Research Workshop, 145-148. doi:10.7152/acro.v11i1.12778

\title{
Interfaces for understanding: Improving access to consumer health information
}

This research will examine how health care consumers use a prototype interface that supports understanding and defining information problems through visual exploration of the medical domain (relationships among medical concepts and functional relationships in the human body).

\section{Description}

The majority of health care consumers must overcome a number of obstacles when conducting searches within medical databases. Because many consumers are unfamiliar with specific medical terminology or knowledge about the medical domain, the underlying mental representation of their information problem is inadequate for formulating queries, making decisions concerning the relevance of materials, and extracting needed information from the retrieved materials. The goal of this research is to investigate the use of interface visualization tools to support the dynamic cognitive process of information seeking.

\section{Overall Context}

The current trend in health care is to promote active consumer participation in matters related to personal health decisions and concerns. Consumer information enables people to take control of their own health or the health of a family member, offering a number of benefits including "more truly informed consent, increased patient understanding and satisfaction, increased compliance with treatment, and quicker, less stressful recovery from illness and surgery" (Gann, 1995). As a result of increased demand, there has been an "explosion" of medical information available on the Internet that is targeted towards consumers. However, the queries that users are able to enter into medical search systems often result in frustration and disappointment (McCray et al., 1999; Tolle et al., 2000).

During the initial stages of the search process, users often struggle to articulate an information need based on an incomplete or inaccurate mental representation of their actual information requirements (Taylor, 1962). Many searches fail because the user could not verbalize his/her information need in order to formulate a sufficient query. Even if the user receives a set of relevant materials, the search may still be deemed unsuccessful if the user is unable to judge whether the returned materials contain the information s/he is seeking or cannot comprehend the content. In some situations, information professionals can assist users in understanding and clarifying ambiguous information needs, selecting appropriate resources, and guiding the user toward an acceptable outcome (White, 1998). However, in an age where a large number of searches are conducted without the assistance of a professional intermediary, such as through the Internet, many users are left stranded.

Providing visual exploration and browsing mechanisms within the search interface would be useful to consumers using health information systems. Interfaces that allow users to visualize conceptual and functional relationships are "external aids" that can enhance cognitive abilities (Card et al., 1999; Pinto et al., 1997; Jonassen et al., 1997). Interactive visualizations of the medical domain concept space (e.g., MeSH) may assist users in 1) clarifying their information need and formulating queries, 2) making decisions concerning the relevance of materials, and 3 ) 
Slaughter, . (2000). Interfaces for understanding: Improving access to consumer health information. . 11th ASIS SIG/CR Classification Research Workshop, 145-148. doi:10.7152/acro.v11i1.12778

understanding and extracting information contained in the retrieved materials. Consider, for instance, a user who has just been diagnosed with Parkinson's disease and would like to learn about the progression of the disease. A method of displaying the interrelation between dopamine and Parkinson's disease would greatly improve the user's ability to formulate his/her query. Relationships such as the interactions between body systems, diseases, and treatments displayed together in connection with materials in the database can not only provide overviews but also permit the user to get a better understanding of underlying concepts.

\section{Proposed Research}

Defining and understanding the information need problem is a critical step that remains active throughout the information seeking process and is, unfortunately, either assumed or abbreviated in end-user search systems (Marchionini, 1995). This research will study the ways in which the information seeking process is aided by an interface designed to augment consumers' abilities to formulate queries, make relevance decisions and understand concepts in retrieved materials.

\section{1 Research Questions}

Q1. What kinds of medical information do health care consumers need and where do they obtain this information in order to understand, define and verbalize their information need?

Q2. What interface features are most effective for facilitating an understanding of conceptual and functional relationships?

Q3. How do consumers use the visualization tools in the prototype interface?

Q4. How do new methods for browsing medical concepts impact query formulation, relevance decisions, and material use?

\subsection{Methodology}

Due to the exploratory nature of this work, a qualitative methodology will be used. A specific medical domain will be selected. The work will be divided into two phases.

\subsubsection{Phase 1: Designing/building the database and user interface for Phase 2. (Focus on research question 1)}

1. Literature review of consumer health information needs and use studies

2. Focus group data collected from health care consumer participants

3. Develop an underlying database of connected medical concepts

4. Guided by focus group data and principles derived from information needs and use studies, design and develop the user interface

\subsubsection{Phase 2: Qualitative study (Focus on research questions 2,3, and 4)}

1. 5-10 consumers as participants 
Slaughter, . (2000). Interfaces for understanding: Improving access to consumer health information. . 11th ASIS SIG/CR Classification Research Workshop, 145-148. doi:10.7152/acro.v11i1.12778

2. Observation of participants using the prototype interface as they explore a real information problem of their own choice, using a think-aloud protocol

3. Interviews with participants prior to and following observation session

4. Analysis of search-related materials (example: search history log)

5. Elicitation of participants understanding of conceptual and functional relationships (Jonassen, 1997)

\section{References}

Card, S., Mackinlay, J., \& Shneiderman, B. (1999). Readings in Information Visualization: Using Vision to Think. Morgan Kaufman Publishers: San Francisco.

Gann, R.(1995). Consumer health information: information for the public, patients and carers. In Michael Carmel (Ed.) Health Care: Librarianship and Information Work, $2^{\text {nd }}$ edition (pp.59-71). Library Association Publishing: London.

Jonassen, D. H., Reeves, Th .C., Hong, N., Harvey, D. and Peters, K. (1997). Concept mapping as cognitive learning and assessment tools. J. of Interactive Learning Res., 8(3/4), 289-308.

Marchionini, G. (1995). Information seeking in electronic environments. Cambridge Series on Human Computer Interaction. Cambridge University Press: New York.

McCray, A. T., Loane, R. F., Browne, A .C., and Bangalore A. K. (1999). Terminology issues in user access to Web-based medical information. Proc AMIA Symp 1999, 107-111.

Pinto, A. J. and Zeitz, H. J. (1997). Concept mapping: a strategy for promoting meaningful learning in medical education. Medical Teacher, 19(2), 114-121.

Taylor, R. (1962). The process of asking questions. American Documentation, 13, 391-397.

Tolle, K. \& Chen, H. (2000). Comparing Noun Phrasing Techniques for Use with Medical Digital Library Tools. Journal of the American Society for Information Science (JASIS), 51(4), $352-370$.

White, M .D. (1998). Questions in reference interviews. Journal of Documentation, 54 (September), 443-465. 


\section{Questions}

1. Does the use of a visual representation of a knowledge structure help users to see relationships between concepts, clear up faulty views and construe meaning from context? How can a visual representation of a thesaurus aid a user in query formulation?

2. What kinds of visual representations might be made using a thesaurus? How would users interact with these visual thesaurus interfaces?

3. How can the use of a visual thesaurus interface be evaluated? 